\title{
Spontaneous remission of fully symptomatic visceral leishmaniasis
}

Oussama Mouri $^{{ }^{*}+}$, Mathilde Benhamou ${ }^{5 \dagger}$, Gaëlle Leroux ${ }^{2}$, Nathalie Chartrel ${ }^{1}$, Alain Devidas ${ }^{4}$, Marc Thellier ${ }^{1,6}$, Zahir Amoura ${ }^{2}$, Nathalie Costedoat-Chalumeau ${ }^{3+}$ and Pierre Buffet ${ }^{1,6+}$

\begin{abstract}
Background: Visceral leishmaniasis (VL), i.e., infection with Leishmania sp. associated with high fever, weight loss, massive splenomegaly and markedly altered laboratory parameters, is generally fatal if untreated. The possibility of transient spontaneous remission of fully symptomatic visceral leishmaniasis (VL) has been mentioned but, to our knowledge) has never been documented.

Case presentation: We report the first documented history of a patient with overt, confirmed VL experiencing a complete remission in the absence of any anti-leishmanial therapy. The diagnosis of $V L$ at the time of the self-resolving episode was strongly suspected based on clinical presentation and presence of antileishmanial antibody, then unequivocally confirmed years later by the presence of an amastigote on a stored smear and the positive quantitative PCR with Leishmania-specific primers from the material scraped from this same slide

Conclusion: This report demonstrates that complete spontaneous remission may occur in patients with overt, fully symptomatic VL. VL should therefore be considered in cases of self-resolving or relapsing episodes of fever of unknown origin. Confirmation should be based on both serological tests and specific PCR on a blood sample.
\end{abstract}

Keywords: Visceral leishmaniasis, Spontaneous remission, Hemophagocytic lymphohistiocytosis, Real time PCR, Giemsa stained, Bone marrow smear

\section{Background}

Overt visceral leishmaniasis, i.e., infection with Leishmania sp. associated with high fever, weight loss, massive splenomegaly and markedly altered laboratory parameters, is generally considered fatal if untreated $[1,2]$. The possibility of transient spontaneous remission has been mentioned [3] but, to our knowledge, has never been precisely documented because patients with documented VL are treated. We provide the first description of a complete, prolonged remission of fully symptomatic VL in a patient who had not received any anti-leishmanial therapy.

\section{Case presentation}

In July 2008, a 27-year-old immunocompetent male patient was referred to our hospital for a 3-week history of fever, asthenia and pancytopenia. The patient had spent

\footnotetext{
* Correspondence: oussama.mouri@psl.aphp.fr

${ }^{+}$Equal contributors

'AP-HP, Hôpital Pitié-Salpêtrière, Service de Parasitologie, F-75013 Paris, France

Full list of author information is available at the end of the article
}

4 month in an area endemic for Leishmania infantum in the South of France (Oriental Pyrennees). His medical history included dental infections and an allergy to methicillin. He reported two similar episodes of high fever and asthenia in 2006 (Table 1). The first episode in June 2006 had resolved spontaneously. During the second episode, in November, the patient was hospitalized with splenomegaly $(17 \mathrm{~cm})$, fever $\left(40{ }^{\circ} \mathrm{C}\right)$ and weight loss $(10 \mathrm{~kg})$, anemia, leucopenia and thrombocytopenia (Table 1). Microscopic examination of a bone marrow (BM) aspirate was negative for parasites. The bone marrow smear showed normal cellularity and there was no indication of immunosuppression. All microbiological and immunological tests were negative (blood and urine culture, testing for HIV, salmonella, brucellosis, Lyme borreliosis, hepatitis A, cytomegalovirus infection, arboviral infection, and malaria) except for positive titers of anti-Leishmania antibody (IFA 1/400) and a positive serology for EBV suggestive of prior infection. In this context of fever of unknown origin, a presumptive 8-day course of intravenous antibiotic therapy (ceftriaxone and 
Table 1

\begin{tabular}{|c|c|c|c|c|c|c|}
\hline & 2006 Nov. 13th & 2007, Feb. 1st & 2008, July 2nd & 2008 , July 11 th & 2008, July 21st & 2009, Jan. 15th \\
\hline Antileishmanial agent & & & & L-AmB Day1 & L-AmB Day10 & \\
\hline Antimicrobial agents & Ciprofloxacin ceftriaxone & & & & & \\
\hline \multicolumn{7}{|l|}{ Clinical parameters } \\
\hline Temperature $\left({ }^{\circ} \mathrm{C}\right)$ & 40 & 37 & 40 & 38.2 & 36.1 & 36.5 \\
\hline Weight (kg) & 59 & 73 & 68 & & & 72 \\
\hline \multicolumn{7}{|l|}{ Biological parameters } \\
\hline White blood cell count (/mL) & 1.480 & 7.000 & 1.280 & 0.990 & 4.110 & 9.200 \\
\hline Neutrophils (/mL) & 0.720 & 4.018 & 0.563 & 0.495 & 1.993 & 6.522 \\
\hline Lymphocytes (/mL) & 0.650 & 2.065 & 0.640 & 0.455 & 1.780 & 1.536 \\
\hline Hemoglobin (g/dL) & 9.6 & 14.8 & 9.9 & 9.1 & 11.6 & 16.2 \\
\hline Platelet count (/mL) & 87.000 & 197.000 & 75.000 & 64.000 & 250.000 & 182.000 \\
\hline ASAT (IU/L) & 126 & 21 & 51 & 93 & & 20 \\
\hline ALAT (IU/L) & 56 & 18 & 15 & 19 & & 17 \\
\hline C-reactive protein (mg/L) & 160 & 1.9 & 63 & 97 & 4 & \\
\hline Ferritin $(\mu \mathrm{g} / \mathrm{L})$ & 19.295 & 57 & 2.093 & & & \\
\hline Fibrinogen (g/l) & 2.5 & 3.5 & 3.5 & & & \\
\hline Triglycerides (mmol/L) & 2.32 & & 2.21 & & & \\
\hline LDH (IU/L) & 1.400 & 250 & 1.010 & & & \\
\hline
\end{tabular}

ofloxacin) was administered. All signs and symptoms, including fever, splenomegaly and asthenia, resolved in 4 weeks. No specific anti-leishmanial drug had been administered, as the treating physician had not retained this diagnosis. All laboratory parameters normalized during the same period, confirming complete remission of the episode (Table 1).

In 2008, relapse occurred with weight loss $(4 \mathrm{~kg})$, fever $\left(40{ }^{\circ} \mathrm{C}\right.$ ) and arthralgia (Table 1$)$. Physical examination showed an enlarged spleen, measuring $20 \mathrm{~cm}$ on the subsequent CT scan. Antinuclear antibodies were positive (dilution of 1/1280) with a nonspecific pattern. The bone marrow (BM) aspirate showed hemophagocytosis. All microbiological tests, including Histoplasma capsulatum antibody were negative except anti-leishmanial antibody titers that were again positive (ELISA $>1.7$ for a threshold at 1). Quantitative polymerase chain reaction (PCR) on a L. infantum kinetoplast DNA target found 30 parasites per $\mathrm{mL}$, i.e., above the threshold for active VL (1 parasite/ml) [4]. Blood and BM cultures for $L$. infantum were negative.

Fever lasted 4 weeks before the diagnosis of VL was confirmed. The patient was then treated with liposomal Amphotericin B (l-AmB) (3 mg/kg/day D1-5 and D10) [5]. Complete fever resolution was obtained in 3 days along with a dramatic improvement of the patient's general well-being. Ultrasonography showed regression of splenomegaly from 20 to $14 \mathrm{~cm}$ at the end of the first week, followed by normalization to $12 \mathrm{~cm} 6$ months later. At that time laboratory parameters had returned to normal levels (Table 1) and quantitative PCR for $L$. infantum was negative. When last seen, in September 2010, the patient was completely asymptomatic.

We reexamined a bone marrow smear performed in 2006 during the first hospitalization. Independent, complete examination of this smear by 2 observers (10 h of observation under the microscope) revealed the presence of a single typical amastigote form of Leishmania sp. (Fig. 1). Material on the smear was then thoroughly scarped from the slide with a blade and resuspended in $30 \mu \mathrm{l}$ of sterile distilled water. Real time PCR amplification was performed in a thermal cycler TAQMAN ${ }^{\circledR}$ (Applied Biosystems) using Leishmania kDNA specific primers as described by Mary et al. [4]. One negative blood slide and one negative tissue slide were used as controls. Amplification of the material retrieved from the slide occurred at $28 \mathrm{CTs}$, corresponding to the presence of 1.8 parasites (Fig. 1).

\section{Discussion}

This is the first documented report of a patient with overt, confirmed VL experiencing a complete remission in the absence of any anti-leishmanial therapy. The diagnosis of VL at the time of the self-resolving episode was strongly suspected based on clinical presentation and presence of antileishmanial antibody, then unequivocally confirmed years later by the presence of an amastigote on a stored smear. Quantitative PCR from the material scraped from this same slide using Leishmania-specific primers was positive. The excellent specificity of this 


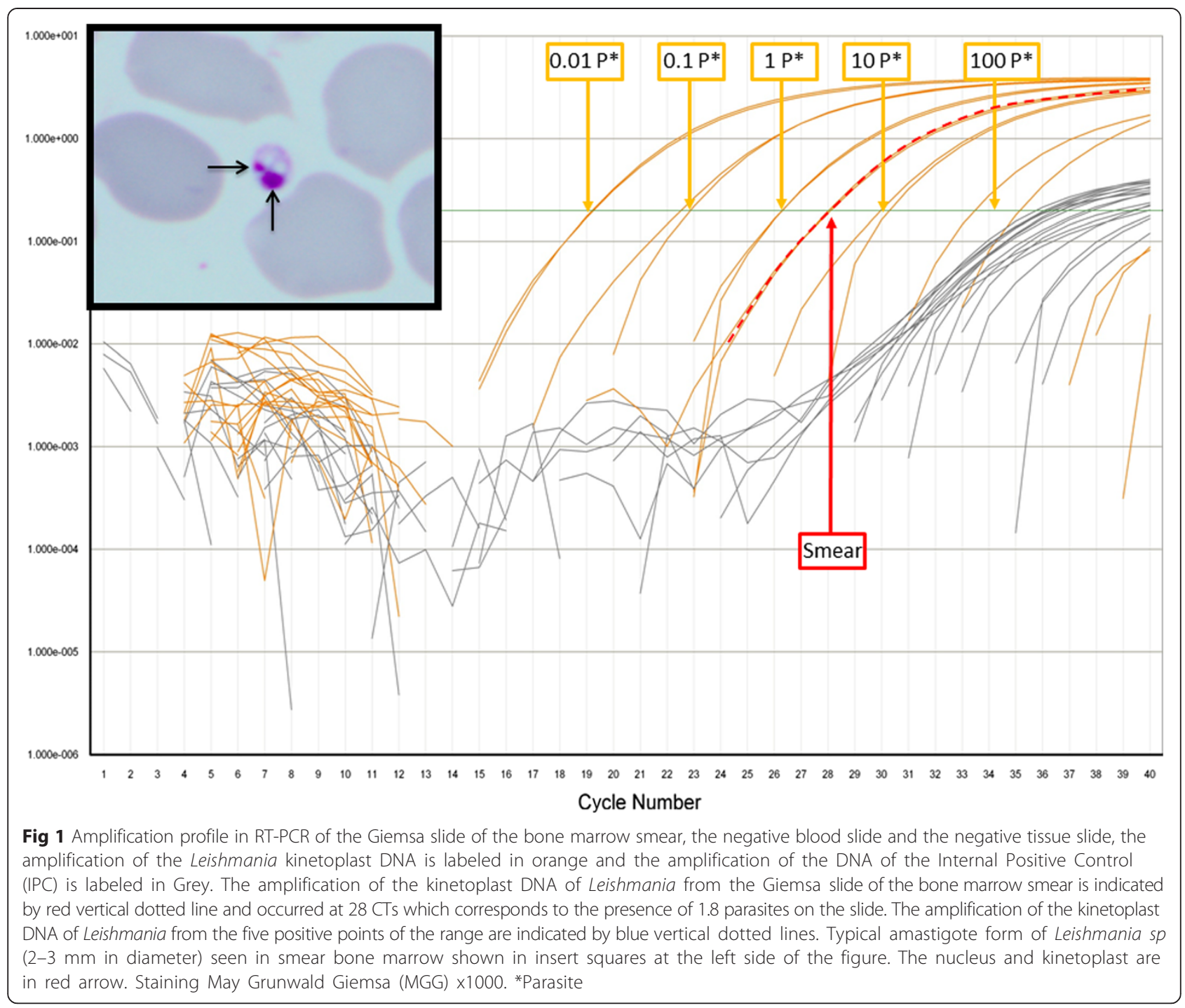

PCR has been confirmed by several teams [4, 6-9]. The last episode, during which PCR was positive in a blood sample was followed by a positive outcome under antileishmanial therapy with liposomal amphotericin B. No relapse occurred during the 2 years following administration of anti-leishmanial therapy, which is consistent with the high efficacy of l-AmB in Mediterranean VL [10].

It has been known for decades that patients with mild symptoms of VL may recover spontaneously and that a minority eventually develop overt clinical VL [11]. In $L$. infantum transmission foci, asymptomatic infections and mild clinical forms of $\mathrm{VL}$ are indeed frequent in humans $[12,13]$. This is different from the spontaneous remission of fully symptomatic severe VL, as described here. Uncontrolled studies that show improvement or apparent cure in patients receiving experimental anti-leishmanial interventions for overt VL should thus be interpreted cautiously $[14,15]$. We cannot definitely exclude the hypothesis that the short course of intravenous antibiotic therapy (ceftriaxone and ofloxacin during 8 days) during the first episode may have contributed to the patient's improvement. A few reports in vitro or in animals suggest indeed that quinolones may have some activity against Leishmania [16-18] thus possibly contributing to the control of parasite loads. Thereafter, the patient was appropriately treated and cured with liposomal Amphotericin B.

For confirmation of VL, PCR in the blood is more sensitive than conventional search of the parasite even in bone marrow aspirates. Time spent for reading Giemsa-stained smears in the search for Leishmania amastigotes increases the test sensitivity that raises to $95.4 \%$ after $60 \mathrm{~min}$ and $89.7 \%$ when 1.200 fields are examined [19]. When a macrophage activation syndrome is present, repetition of bone marrow aspirates also increases diagnostic sensitivity [20]. Why patients would spontaneously recover from overt VL is not clear. Interestingly, our patient met five out 
of the eight criteria of the hemophagocytic lymphohistiocytosis (HLH-2004 revised diagnostic guidelines) [21]. Therefore, if HLH had also occurred during the initial episode, phagocytosis of Leishmania-infected cells by previously activated macrophages, or a general activation of macrophages as observed in HLH, may have contributed to the control of parasite loads. This hypothesis is consistent with the absence of Leishmania amastigotes in bone marrow aspirates in $36.3 \%$ of patients with VL and HLH [22].

\section{Conclusion}

In summary, this report demonstrates that complete spontaneous remission may occur in patients with overt, fully symptomatic VL. VL should therefore be considered in cases of self-resolving or relapsing episodes of fever of unknown origin. Confirmation should be based on both serological tests and specific PCR on a blood sample.

\section{Consent}

Patient was informed of the process by this attending physician using a procedure common to all French National Reference Centers (NRC) (http://www.parasitologie.univmontp1.fr/conseil.htm) and gave his oral consent for data collection and publication. Mention of this consent was written in the medical chart.

\section{Abbreviations \\ VL: Visceral leishmaniasis; PCR: Polymerase chain reaction; HIV: Human immunodeficiency virus; EBV: Epstein-barr virus; HLH: Hemophagocytic lymphohistiocytosis.}

\section{Competing interests}

The authors declare that they have no competing interests.

\section{Authors' contributions}

PB NCC OM ZA AD have made the diagnosis and contributed to patient care. PB ZA GL AD have designed the analysis. PB OM NCC MB MT wrote the paper. NC MT have contributed vital samples/information/materials/tools. All authors read and approved the final version.

\section{Acknowledgements}

We thank nurses and technicians from the medical department and the clinical laboratory for their outstanding contribution to the management of patient. This work was not supported by any external funding.

\section{Author details}

${ }^{1}$ AP-HP, Hôpital Pitié-Salpêtrière, Service de Parasitologie, F-75013 Paris, France. ${ }^{2}$ AP-HP, Hôpital Pitié-Salpêtrière, Service de Médecine Interne, F-75013 Paris, France. ${ }^{3}$ AP-HP, Hôpital Cochin, Service de Médecine Interne. Centre de référence maladies auto-immunes et systémiques rares, Service de Médecine Interne, F-75014 Paris, France. ${ }^{4}$ Hopital Sud Francilien, Service d'Hématologie, Corbeil-Essonnes, France. ${ }^{5}$ AP-HP, Hôpital Cochin, Service de Rééducation et Réadaptation, F-75014 Paris, France. ' UPMC Université Paris 06, Centre Immunologie et Maladies Infectieuses, F-75005 Paris, France.

Received: 14 April 2015 Accepted: 7 October 2015

Published online: 23 October 2015

\section{References}

1. Pearson R, de Queiroz Sousa A, Jeronimo SB. Leishmania species: visceral (KalaAzar), cutaneous and mucosal leishmaniasis. In: Mandell GL, Bennett JE, Dolin R, editors. Mandell, Douglas, and Bennett's Principles and practice of infectious diseases. Volume 2. Philadelphia: Churchill Linvingstone; 2000. p. 2831-45.
2. den Boer ML, Alvar J, Davidson RN, Ritmeijer K, Balasegaram M. Developments in the treatment of visceral leishmaniasis. Expert Opin Emerg Drugs. 2009;14(3):395-410.

3. Bern C, Maguire JH, Alvar J. Complexities of assessing the disease burden attributable to leishmaniasis. PLoS Negl Trop Dis. 2008;2(10), e313.

4. Mary C, Faraut F, Lascombe L, Dumon H. Quantification of Leishmania infantum DNA by a real-time PCR assay with high sensitivity. J Clin Microbiol. 2004:42(11):5249-55.

5. Davidson RN, di Martino L, Gradoni L, Giacchino R, Gaeta GB, Pempinello R, et al. Short-course treatment of visceral leishmaniasis with liposomal amphotericin B (AmBisome). Clin Infect Dis. 1996;22(6):938-43.

6. Brustoloni YM, Lima RB, da Cunha RV, Dorval ME, Oshiro ET, de Oliveira $A L$, et al. Sensitivity and specificity of polymerase chain reaction in Giemsa-stained slides for diagnosis of visceral leishmaniasis in children. Mem Inst Oswaldo Cruz. 2007;102(4):497-500.

7. Fisa R, Riera C, Lopez-Chejade P, Molina I, Gallego M, Falco V, et al. Leishmania infantum DNA detection in urine from patients with visceral leishmaniasis and after treatment control. Am J Trop Med Hyg. 2008;78(5):741-4.

8. Ozerdem D, Eroglu F, Genc A, Demirkazik M, Koltas IS. Comparison of microscopic examination, rK39, and PCR for visceral leishmaniasis diagnosis in Turkey. Parasitol Res. 2009;106(1):197-200.

9. Fraga TL, Brustoloni YM, Lima RB, Dorval ME, Oshiro ET, Oliveira J, et al. Polymerase chain reaction of peripheral blood as a tool for the diagnosis of visceral leishmaniasis in children. Mem Inst Oswaldo Cruz. 2010;105(3):310-3.

10. Gradoni L, Soteriadou K, Louzir H, Dakkak A, Toz SO, Jaffe C, et al. Drug regimens for visceral leishmaniasis in Mediterranean countries. Trop Med Int Health. 2008;13(10):1272-6.

11. Evans TG, Teixeira MJ, Sousa Ade Q, Pearson RD. Short report: extended follow-up of the natural history of persons infected with Leishmania chagasi. Am J Trop Med Hyg. 1995;53(4):360-1.

12. Badaro R, Jones TC, Carvalho EM, Sampaio D, Reed SG, Barral A, et al. New perspectives on a subclinical form of visceral leishmaniasis. J Infect Dis. 1986;154(6):1003-11.

13. Jeronimo SM, Teixeira MJ, Sousa A, Thielking P, Pearson RD, Evans TG. Natural history of Leishmania (Leishmania) chagasi infection in Northeastern Brazil: long-term follow-up. Clin Infect Dis. 2000;30(3):608-9.

14. Jha TK. Evaluation of diamidine compound (pentamidine isethionate) in the treatment resistant cases of kala-azar occurring in North Bihar, India. Trans R Soc Trop Med Hyg. 1983;77(2):167-70.

15. Sundar S, Murray HW. Gamma interferon in the treatment of Kala-azar and other forms of Leishmaniasis. J Assoc Physicians India. 1995;43(5):348-50.

16. Romero IC, Saravia NG, Walker J. Selective action of fluoroquinolones against intracellular amastigotes of Leishmania (Viannia) panamensis in vitro. J Parasitol. 2005;91(6):1474-9.

17. Bianciardi P, Fasanella A, Foglia Manzillo V, Trotta T, Pagano A, Sorino S, et al. The efficacy of enrofloxacin, alone or combined with metronidazole, in the therapy of canine leishmaniasis. Parasitol Res. 2004;93(6):486-92.

18. Cortazar TM, Coombs GH, Walker J. Leishmania panamensis: comparative inhibition of nuclear DNA topoisomerase II enzymes from promastigotes and human macrophages reveals anti-parasite selectivity of fluoroquinolones, flavonoids and pentamidine. Exp Parasitol. 2007;116(4):475-82.

19. da Silva MR, Stewart JM, Costa CH. Sensitivity of bone marrow aspirates in the diagnosis of visceral leishmaniasis. Am J Trop Med Hyg. 2005;72(6):811-4.

20. Gagnaire MH, Galambrun C, Stephan JL. Hemophagocytic syndrome: A misleading complication of visceral leishmaniasis in children-a series of 12 cases. Pediatrics. 2000;106(4), E58.

21. Henter Jl, Horne A, Arico M, Egeler RM, Filipovich AH, Imashuku S, et al. HLH-2004: Diagnostic and therapeutic guidelines for hemophagocytic lymphohistiocytosis. Pediatr Blood Cancer. 2007;48(2):124-31.

22. Rajagopala S, Dutta U, Chandra KS, Bhatia P, Varma N, Kochhar R. Visceral leishmaniasis associated hemophagocytic lymphohistiocytosis-case report and systematic review. J Infect. 2008;56(5):381-8. 